\title{
Positive association between increased popliteal artery vessel wall thickness and generalized osteoarthritis: is OA also part of the metabolic syndrome?
}

\author{
Peter R. Kornaat • Ruby Sharma - Rob J. van der Geest • Hildo J. Lamb • \\ Margreet Kloppenburg • Marie-Pierre Hellio le Graverand • Johan L. Bloem • \\ Iain Watt
}

Received: 7 April 2009 / Accepted: 7 June 2009 / Published online: 3 July 2009

(C) The Author(s) 2009. This article is published with open access at Springerlink.com

\begin{abstract}
Purpose The purpose of the study was to determine if a positive association exists between arterial vessel wall thickness and generalized osteoarthritis (OA). Our hypothesis is that generalized $\mathrm{OA}$ is another facet of the metabolic syndrome.

Materials and methods The medical ethical review board of our institution approved the study. Written informed consent was obtained from each patient prior to the study. Magnetic resonance (MR) images of the knee were obtained in 42 patients who had been diagnosed with generalized OA at multiple joint sites. Another $27 \mathrm{MR}$ images of the knee were obtained from a matched normal (non-OA) reference population. Vessel wall thickness of the popliteal artery was quantitatively measured by dedicated software. Linear regression models were used to investigate the association between vessel wall thickness and generalized OA. Adjustments were made for age, sex, and body mass index (BMI).
\end{abstract}

Pfizer Inc., Groton, CT, USA provided financial support for this work; however, authors who are not employees of Pfizer Inc. had control of the inclusion of all data and information that might present a conflict of interest.

P. R. Kornaat $(\triangle) \cdot$ R. Sharma $\cdot$ R. J. van der Geest $\cdot$

H. J. Lamb J. L. Bloem • I. Watt

Department of Radiology, Leiden University Medical Center,

Albinusdreef 2,

NL-2333 ZA Leiden, The Netherlands

e-mail: P.R.Kornaat@lumc.nl

M. Kloppenburg

Department of Rheumatology, Leiden University Medical Center,

Leiden, The Netherlands

M.-P. Hellio le Graverand

Pfizer Global Research and Development,

50 Pequot Ave,

New London, CT 06320, USA
Confidence intervals (CI) were computed at the $95 \%$ level and a significance level of $\alpha=0.05$ was used.

Results Patients in the generalized OA population had a significant higher average vessel wall thickness than persons from the normal reference population $(p \leq \alpha)$, even when correction was made for sex, age, and BMI. The average vessel wall thickness of the popliteal artery was $1.09 \mathrm{~mm}$ in patients with generalized OA, and $0.96 \mathrm{~mm}$ in the matched normal reference population.

Conclusion The association found between increased popliteal artery vessel wall thickness and generalized osteoarthritis suggests that generalized OA might be another facet of the metabolic syndrome.

Keywords Osteoarthritis · Popliteal artery · Metabolic syndrome $\cdot$ Magnetic resonance imaging

\section{Introduction}

Osteoarthritis (OA) is a chronic, progressive joint disease, leading to pain and loss of function in a considerable proportion of patients, with great impact and consequences in the ageing population of the industrialized world [1]. Known risk factors for hip and knee OA are age, female gender, and obesity [2]. Paradoxically, obesity is also a risk factor for hand OA [3-6]. Whilst the increased joint stress accompanying obesity may explain the strong linkage between obesity and knee and hip OA, it does not explain why hand OA should be linked with obesity. Furthermore, $\mathrm{OA}$ is also associated with an increase in comorbid risk factors including cardiovascular disease and hypertension [7-9]. Since obesity, hypertension, and cardiovascular disease are part of the metabolic syndrome one might wonder if $\mathrm{OA}$ is another aspect of the metabolic syndrome. 
The metabolic syndrome is the concurrence in an individual of multiple metabolic abnormalities associated with atherosclerotic cardiovascular disease. It includes a combination of risk determinants including atherogenic dyslipidemia, elevated blood pressure, elevated blood glucose, a prothrombotic state, and a proinflammatory state [10-12]. Recent guidelines propose that the finding of any three of five easily recognized components (abdominal obesity, elevated triglycerides, reduced high-density lipoprotein (HDL), elevated blood pressure, and elevated blood glucose) are sufficient for a diagnosis of metabolic syndrome in clinical practice $[12,13]$. Furthermore, individuals with metabolic syndrome have a higher frequency of carotid plaques and a higher vessel wall thickness of the common carotid artery $[14,15]$.

Therefore, the hypothesis was that a positive association between arterial vessel wall thickness and generalized OA might suggest that generalized $\mathrm{OA}$ is indeed part of the metabolic syndrome. In the present study we semiautomatically assessed popliteal artery vessel wall thickness in a normal reference population and in 42 patients with generalized OA.

\section{Patients and methods}

\section{Patients}

The present study is part of the ongoing GARP (Genetics, Osteoarthritis and Progression) study [16]. The primary goal of the GARP study is the identification of genetic susceptibility determinants to OA and disease progression in middle-aged sib pairs with OA at multiple joint sites. Patients were required to have symptomatic $\mathrm{OA}$ in at least two or more of the following joint sites: hands, spine (cervical or lumbar), knees or hips. MR image sets of the knee were obtained at study entry [17]. Patients included in the present study were the first 42 consecutive patients from the GARP progression study. The normal reference population consists of volunteers with a sex and age distribution matched to the GARP population. To be included participants had to have minimal radiological spine and radiological hand OA, no clinical signs of OA, like joint pain or stiffness, and no signs of $\mathrm{OA}$ as assessed by physical examination in the hands, hips, spine, and knees. During physical examination, the presence of knee joint effusion, range of motion, malalignment, and joint pain on movement were assessed. Furthermore, they had to have no history of trauma to the knees or familial predisposition to OA, and grade 0 on the Kellgren-Lawrence scale on the knee and hip $\mathrm{X}$-rays. Complete written informed consent was obtained from each patient prior to the study. The medical ethical review board of our institution approved the study.
MR acquisition

For the GARP study, knees were imaged using a transmitreceive 4-channel knee coil in a $1.5 \mathrm{~T}$ superconducting magnet (Philips Medical Systems, Best, the Netherlands). For the retrospective analysis of the current study we selected one sequence to assess the wall of the popliteal artery. We used a fat-suppressed dual turbo spin echo sequence (TR 2,500; TE 7.1/40; NEX 2; 2-mm slice thickness; no gap; $180-\mathrm{mm}$ field of view; $256 \times 256$ acquisition matrix, 62 slices) to assess the vessel wall thickness of the popliteal artery.

Semi-automatic vessel wall thickness quantification

We analyzed all of the images using the VesselMASS software package developed at our institution [18]. This software package allows the semi-automated detection of the luminal and outer wall boundaries of the vessel wall from MR images, and the subsequent derivation of various quantitative parameters describing the vessel wall (Fig. 1). The VesselMass software package has demonstrated excellent agreement between automatic and manual area measurements for the lumen and outer vessel wall contour. We calculated the vessel wall thickness by subtracting the luminal area from the outer contour area. The average vessel wall thickness was calculated by averaging the vessel wall thickness of all slices [18].

\section{Statistical analysis}

The difference in arterial vessel wall thickness between OA patients and the normal reference population was calculated by linear regression models in SPSS for Windows, version 12.0 (SPSS, Chicago, IL, USA). Adjustments were made for age, sex, and body mass index (BMI). Confidence intervals (CI) were computed at the $95 \%$ level and a significance level of $\alpha=0.05$ was used.

\section{Results}

The basic characteristics of the 42 patients with generalized $\mathrm{OA}$ and the 27 controls from the normal reference population are shown in Table 1. Patients in the generalized OA population had a higher average vessel wall thickness than persons from the normal reference population $(p \leq \alpha$, CI: 0.9-2.2), when adjustments were made for sex, age, and BMI. We found a positive association between average vessel wall thickness and age ( $p \leq \alpha$, CI: $0.2-0.6$ ); older people having a significantly higher average vessel wall thickness in both the generalized OA and in the normal reference populations. Patients with generalized OA had a 
Fig. 1 a Axial fat-suppressed spin echo image of the knee after semi-automatic vessel wall thickness quantification in a patient with generalized OA Note the osteophytes at the femoral condyle. b Detail of the semi-automatic vessel wall thickness quantification of the luminal and outer wall boundaries of the vessel wall
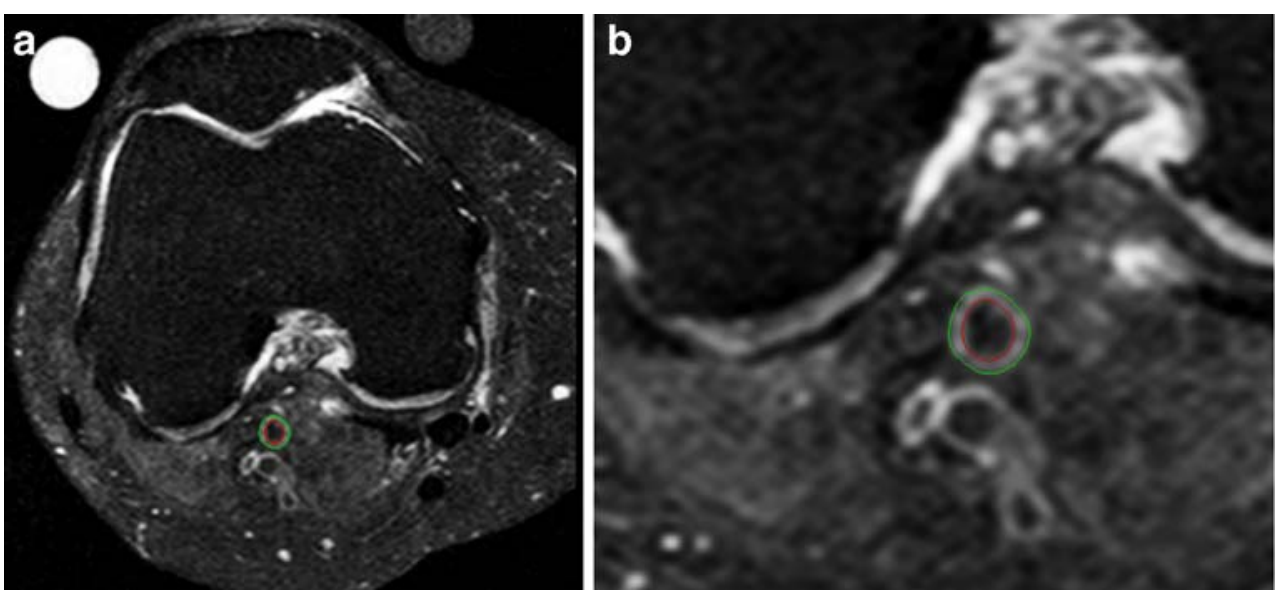

slightly higher BMI than the normal reference population; however, this was not significant ( $p=0.63 \mathrm{CI}$ : -0.4 to 0.2 ). Patients with generalized $\mathrm{OA}$ also showed a slightly decreased hip-waist ratio compared with the normal reference population. However, this too was not significant $(p=0.32$, CI: -0.3 to 0.4$)$.

\section{Discussion}

In the present study, patients with generalized OA had a significantly higher average popliteal artery wall thickness than persons from the normal reference population, even when correction was made for confounders including sex, age, and BMI. Thus, we confirmed an association between a possible feature of the metabolic syndrome, that is, an increased popliteal artery wall thickness, and generalized OA. This is clearly of major significance in the etiology and management of generalized OA. However, caution must be exercised for whilst it has been demonstrated that individuals with metabolic syndrome have a higher frequency of carotid plaques and a higher intima media thickness of the common carotid artery $[14,15]$, data on popliteal artery thickness have not been presented previously and require confirmation. Furthermore, it is not known whether the increased wall thickness of the carotid artery is a localized or generalized medium-sized artery phenomenon. Nonethe- less, the present data do suggest that arterial thickening might be present in generalized $\mathrm{OA}$ and hence may be another sign of the metabolic syndrome. Further, as expected, we confirmed a positive association between average popliteal artery vessel wall thickness and age both in patients with generalized OA and in the normal reference population, and this relationship has been described previously [19]. This too suggests that the data presented here might be accurate and therefore the postulate reasonable.

Recently, two hypotheses were advanced that contribute to a possible causal relationship between obesity-associated metabolic changes and osteoarthritis. The first postulates that obesity-associated atherosclerosis in the subchondral bone might play a role in the progression of osteoarthritis [20]. In osteoarthritis the subchondral bone might be affected by microvascular changes in both the arterial as well as the venous side of the vascular circulation [21] resulting in episodically reduced blood flow through the small vessels in the subchondral bone and finally leading to subchondral ischemia. Vascular disease in subchondral bone may also explain fluctuation in subchondral bone marrow edema lesions, recently reported on MR images in OA patients [22], as it may resemble episodes of avascular necrosis. The link between osteoarthritis and atherosclerosis is supported by epidemiological studies. A higher prevalence of cardiovascular risk factors, like hypertension and
Table 1 Patient characteristics

$\mathrm{OA}=$ osteoarthritis

${ }^{\text {a }}$ Defined as pain or stiffness on most days of the month prior to study entry, in combination with osteophytes on radiographs

\begin{tabular}{lcc}
\hline & Normal reference population & OA population \\
\hline Number of patients & 27 & 42 \\
Age, years, median (range) & $59(44-75)$ & $58(46-75)$ \\
Female sex (\%) & $20(74)$ & $35(83)$ \\
Body mass index $\left(\mathrm{kg} / \mathrm{m}^{2}\right)$, median (range) & $25(20-32)$ & $27(21-35)$ \\
Vessel wall thickness $(\mathrm{mm})$, median (range) & $0.96(0.71-1.10)$ & $1.09(0.96-1.62)$ \\
Hip-waist ratio, median (range) & $1.20(0.95-1.44)$ & $1.17(1.03-1.37)$ \\
Generalized OA, $n(\%)$ & $0(0)$ & $42(100)$ \\
Symptomatic knee OA, $n(\%)^{\mathrm{a}}$ & $0(0)$ & $16(38)$ \\
\hline
\end{tabular}


diabetes type 2, and cardiovascular comorbidity have been suggested in osteoarthritis $[23,24]$. Several studies indicated that cardiovascular mortality may be linked to the severity and extent of osteoarthritis $[25,26]$. In a Finnish cohort it was demonstrated that in men, osteoarthritis in any finger joint predicted eventual cardiovascular death [26].

The second hypothesis postulates that the metabolic changes in the striated muscle induced by the interaction of insulin resistance and systemic inflammation in obese subjects with metabolic syndrome might lead to fatigue and muscle weakness, which negatively influences the balance between damage and repair mechanisms leading to osteoarthritis [27].

The suggestion that $\mathrm{OA}$ is associated, or is part of, the metabolic syndrome may be of clinical relevance and an important finding as it demonstrates another potential etiological factor in the development of more effective treatments to inhibit the progression of OA.

The present study has limitations. First, we associated an indirect sign of the metabolic syndrome, average vessel wall thickness of the popliteal artery, with OA. With the exception of hip-waist ratios, the other direct parameters of the metabolic syndrome (elevated triglycerides, reduced highdensity lipoprotein (HDL), elevated blood pressure, and elevated blood glucose) were not available in the patients studied. Blood samples and blood pressure were taken and recorded, but patients were using medication as necessary. Therefore, standard laboratory/clinical guidelines used to define metabolic syndrome are not used as a gold standard. Second, this was a retrospective pilot study and the findings will need to be confirmed using a larger study population. Third, we have not addressed the apparent paradox between the fact that whilst we found no association between vessel wall thickness and obesity, a positive association between OA and vessel wall thickness was found. However, a definite association exists between OA and obesity and the linkage between the metabolic syndrome is not simply a raised BMI, it is rather associated with intra-abdominal obesity. Hipwaist ratios were available in the present study and showed a trend supporting this concept. A fourth limitation is the absence of an analysis of other potential cofounders such as diet, physical activity, and pain scores. It is possible that patients with generalized OA experienced pain with movement and thus avoid participation in regular physical activity, which may explain in part the vascular pathology. Patients in the generalized OA group did report having more pain in their joints than subjects in the normal reference control group. Lastly, the popliteal artery was analyzed as opposed to the carotid artery. This was a matter of expediency, as the MR imaging available to review included this vessel and it was not considered appropriate to recall patients in order to study either their carotid arteries or thoracic aorta in this retrospective study.
In conclusion, the present study demonstrates a positive association between popliteal artery vessel wall thickness and generalized osteoarthritis, suggesting that generalized OA might also be part of the metabolic syndrome. If this is the case, osteoarthritis might be treated by treating the metabolic syndrome.

Open Access This article is distributed under the terms of the Creative Commons Attribution Noncommercial License which permits any noncommercial use, distribution, and reproduction in any medium, provided the original author(s) and source are credited.

\section{References}

1. Harris ED Jr. The bone and joint decade: a catalyst for progress. Arthritis Rheum. 2001;44:1969-70.

2. Moskowitz RW, Altman RD, Hochberg MC, Buckwalter VM. Risk factors in OA summarized in osteoarthritis, diagnosis and medical/surgical management. 4th ed. Philadelphia: Lippincott Williams \& Wilkins; 2007.

3. Carman WJ, Sowers M, Hawthorne VM, Weissfeld LA. Obesity as a risk factor for osteoarthritis of the hand and wrist: a prospective study. Am J Epidemiol. 1994;139:119-29.

4. Cicuttini FM, Baker JR, Spector TD. The association of obesity with osteoarthritis of the hand and knee in women: a twin study. J Rheumatol. 1996;23:1221-6.

5. Felson DT, Chaisson CE. Understanding the relationship between body weight and osteoarthritis. Baillieres Clin Rheumatol. 1997;11:671-81.

6. Davis MA, Neuhaus JM, Ettinger WH, Mueller WH. Body fat distribution and osteoarthritis. Am J Epidemiol. 1990;132:701-7.

7. Singh G, Miller JD, Lee FH, Pettitt D, Russell MW. Prevalence of cardiovascular disease risk factors among US adults with selfreported osteoarthritis: data from the Third National Health and Nutrition Examination Survey. Am J Manag Care. 2002;8:S383-91.

8. Breedveld FC. Osteoarthritis - the impact of a serious disease. Rheumatology (Oxford). 2004;43(Suppl 1):i4-8.

9. Marks R, Allegrante JP. Comorbid disease profiles of adults with end-stage hip osteoarthritis. Med Sci Monit. 2002;8:CR305-9.

10. Gami AS, Witt BJ, Howard DE, et al. Metabolic syndrome and risk of incident cardiovascular events and death: a systematic review and meta-analysis of longitudinal studies. J Am Coll Cardiol. 2007;49:403-14.

11. Ferrannini E. Metabolic syndrome: a solution in search of a problem. J Clin Endocrinol Metab. 2007;92:396-8.

12. Grundy SM. Metabolic syndrome: a multiplex cardiovascular risk factor. J Clin Endocrinol Metab. 2007;92:399-404.

13. Grundy SM, Cleeman JI, Daniels SR, et al. Diagnosis and management of the metabolic syndrome: an American Heart Association/National Heart, Lung, and Blood Institute Scientific Statement. Circulation. 2005;112:2735-52.

14. Empana JP, Zureik M, Gariepy J, et al. The metabolic syndrome and the carotid artery structure in noninstitutionalized elderly subjects: the three-city study. Stroke. 2007;38:893-9.

15. Iannuzzi A, De MM, Bond MG, et al. Carotid artery remodeling in middle-aged women with the metabolic syndrome (from the "Progetto ATENA" study). Am J Cardiol. 2005;96:1162-5.

16. Riyazi N, Meulenbelt I, Kroon HM, et al. Evidence for familial aggregation of hand, hip, and spine but not knee osteoarthritis in siblings with multiple joint involvement: the GARP study. Ann Rheum Dis. 2005;64:438-43. 
17. Kornaat PR, Bloem JL, Ceulemans RY, et al. Osteoarthritis of the knee: association between clinical features and MR imaging findings. Radiology. 2006;239:811-7.

18. Adame IM, van der Geest RJ, Wasserman BA, Mohamed MA, Reiber JH, Lelieveldt BP. Automatic segmentation and plaque characterization in atherosclerotic carotid artery MR images. MAGMA. 2004;16:227-34.

19. Ebrahim S, Papacosta O, Whincup P, et al. Carotid plaque, intima media thickness, cardiovascular risk factors, and prevalent cardiovascular disease in men and women: the British Regional Heart Study. Stroke. 1999;30:841-50.

20. Conaghan PG, Vanharanta H, Dieppe PA. Is progressive osteoarthritis an atheromatous vascular disease? Ann Rheum Dis. 2005;64:1539-41.

21. Findlay DM. Vascular pathology and osteoarthritis. Rheumatology (Oxford). 2007;46:1763-8.

22. Kornaat PR, Kloppenburg M, Sharma R, et al. Bone marrow edema-like lesions change in volume in the majority of patients with osteoarthritis; associations with clinical features. Eur Radiol. 2007;17:3073-8.
23. Hart DJ, Doyle DV, Spector TD. Association between metabolic factors and knee osteoarthritis in women: the Chingford Study. J Rheumatol. 1995;22:1118-23.

24. Kadam UT, Jordan K, Croft PR. Clinical comorbidity in patients with osteoarthritis: a case-control study of general practice consulters in England and Wales. Ann Rheum Dis. 2004;63: 408-14.

25. Cerhan JR, Wallace RB, el-Khoury GY, Moore TE, Long CR. Decreased survival with increasing prevalence of full-body, radiographically defined osteoarthritis in women. Am J Epidemiol. 1995;141:225-34.

26. Haara MM, Manninen $\mathrm{P}$, Kroger $\mathrm{H}$, et al. Osteoarthritis of finger joints in Finns aged 30 or over: prevalence, determinants, and association with mortality. Ann Rheum Dis. 2003;62: $151-8$.

27. Rojas-Rodriguez J, Escobar-Linares LE, Garcia-Carrasco M, Escarcega RO, Fuentes-Alexandro S, Zamora-Ustaran A. The relationship between the metabolic syndrome and energyutilization deficit in the pathogenesis of obesity-induced osteoarthritis. Med Hypotheses. 2007;69:860-8. 